\title{
Diversidade de dietas na assembléia de linguados (Teleostei, Pleuronectiformes) do manguezal da Baía de Guaratuba, Paraná, Brasil*
}

\author{
Paulo de Tarso da C. Chaves ${ }^{1 * *} \&$ Adriana Serenato \\ Universidade Federal do Paraná, Departamento de Zoologia \\ (Caixa Postal 19020, 81531-990, Curitiba, PR, Brasil) \\ 'e-mail: poissons@cce.ufpr.br
}

- Abstract: Citharichthys arenaceus and C. spilopterus form together almost $70 \%$ of the flatfishes abundance in the mangrove of the Guaratuba Bay. The other important Pleuronectiformes species are Symphurus tessellatus, Etropus crossotus and Achirus lineatus. The main food items of this assemblage are composed by crustacea, fishes, Gastropoda and Polychaeta. None of these species was found to have a highly specialized diet, but they present some particularities: Citharichthys spp and $S$. tessellatus have a diet based on Caridea and fish, E. crossotus based on Amphipoda Gammaridae, and $A$. lineatus based on Polychaeta. The partially overlapping habits correspond to the most generalized diets, so any interspecific competition would be reduced by the nature of the complementary food items. Additional support for this hypothesis is provided by the results concerning both the species density and the percent occurrence of the prey categories.

- Resumo: Citharichthys arenaceus e C. spilopterus são responsáveis por cerca de $70 \%$ da abundância de linguados no manguezal da Baía de Guaratuba. Nesta assembléia, da qual também fazem parte Symphurus tessellatus, Etropus crossotus e Achirus lineatus, a dieta baseia-se em crustáceos, peixes, Gastropoda e Polychaeta. Seu hábito alimentar não é altamente especializado, mas algumas particularidades são observadas: em Citharichthys spp e $S$. tessellatus, Caridea e peixes são os principais itens ingeridos; em $E$. crossotus, Amphipoda Gammaridae; e em $A$. lineatus, Polychaeta. As espécies cuja dieta está em maior grau sobreposta com outras são também aquelas cujo hábito é mais generalista. Em tais casos, a natureza dos itens complementares confere identidade própria a cada dieta, atenuando uma eventual competição interespecífica. Os resultados da associação entre a abundância das populações e o grau de participação dos itens na dieta de cada uma reforçam esta conclusão.

- Descriptors: Pleuronectiformes, Feeding, Mangrove, Brazil.

- Descritores: Pleuronectiformes, Alimentação, Manguezal, Brasil.

(*) Contribuição n.1025 do Departamento de Zoologia da Universidade Federal do Paraná. Trabalho realizado com auxílios do CNPq e da FUNPAR/UFPR.

(**) Bolsista do CNPq. 


\section{Introdução}

No manguezal da Baía de Guaratuba, litoral do Estado do Paraná (2552'S;48 $\left.39^{\prime} \mathrm{W}\right)$, os Pleuronectiformes partilham com os Clupeiformes, Ariidae, Perciformes e Tetraodontiformes a condição de grupos permanentes no sistema. Cinco espécies são registradas de forma regular: Citharichthys arenaceus Evermann \& Marsh, 1900, C. spilopterus Gunther, 1862, Etropus crossotus Jordan \& Gilbert, 1882 (Paralichthyidae), S. tessellatus (Quoy \& Gaimard, 1824) (Cynoglossidae) e A. lineatus (Linnaeus, 1758) (Soleidae). As duas primeiras compõem populações sedentárias na área de mangue (Chaves \& Vendel, 1997), mas sobre as demais não há dados disponíveis a respeito.

Alimentando-se de invertebrados bentônicos- e de peixes, os linguados são considerados essencialmente carnívoros (MacPherson, 1978; Pearcy \& Hancock, 1978; Kawakami \& Amaral, 1983; Toepfer \& Fleeger, 1995; Rodriguez, 1996). Como se tratam de indivíduos que mantêm uma dependência direta do substrato, a simpatria entre populações qualifica diferentes espécies como usuárias em potencial dos mesmos recursos, gerando então a expectativa por atributos que evitem competição interespecífica. No que concerne à alimentação, tais atributos podem ser, dentre outros e de maneira não-exclusiva: a utilização de itens diferentes na dieta, a utilização de itens idênticos mas que não ofereçam restrição de abundância, e o deslocamento dos efetivos populacionais para áreas de menor limitação trófica, em épocas quando tal se faça necessário. Objetivando reconhecer atributos que estejam associados à simpatria das 5 espécies no manguezal de Guaratuba, analisam-se a seguir a abundância relativa e a dieta que cada uma pratica na região.

\section{Material e métodos}

Um total de 788 linguados foi coletado através de arrastos de fundo com portas, realizados mensalmente no manguezal da Baía de Guaratuba entre outubro de 1993 e fevereiro de 1997, exceto maio/95 (Fig. 1). Imediatamente após a captura os exemplares foram transportados sob refrigeração para laboratório, identificados, pesados e medidos (comprimento total). Os estômagos que possuíam algum conteúdo foram fixados em solução de formalina $10 \%$. Sob microscópio, os itens presentes foram identificados $\mathrm{e}$ as informações obtidas quantificadas através de 2 métodos: Freqüência de Ocorrência - F.O. (proporção de indivíduos que apresentavam determinado item) e Contagem de Pontos - C.P. (proporção de pontos ocupados por cada item em uma superficie plana regularmente pontuada). Os resultados provenientes dos 2 métodos foram então conjugados através do Índice de Preponderância - I.P. (Natarajan \& Jhingran, 1961, in Juras \& Yamaguti, 1985), definido como:

$I_{. P}{ }_{i}=\left(F . O_{. i} \times C_{. P . i}\right) \times 100 / \Sigma_{i=1 \rightarrow n}\left(F . O_{i} \times\right.$ C.P. $\left._{i}\right)$,

sendo « $\mathrm{i} »$ cada um dos $\mathrm{n}$ tipos de item encontrados.

Estimativas sobre abundância relativa das populações basearam-se no total de indivíduos coletados e no esforço padronizado (20 minutos mensais de arrasto). Face a limitações técnicas para distinção entre Citharichthys arenaceus e $C$. spilopterus durante a primeira parte do estudo, os dados referentes a essas duas espécies foram tratados de forma conjunta. Ainda pelo mesmo motivo, as análises sobre a composição de sua dieta foram separadas em 2 grupos: até setembro/96, reunindo $C$. arenaceus e $C$. spilopterus, e a partir de outubro/96, considerando-as individualmente. As necessárias ressalvas decorrentes de tais procedimentos serão evocadas no momento oportuno, quando da Discussão.

Com o objetivo de estabelecer um ranking de freqüência de participação dos 9 principais itens alimentares na dieta da assembléia, ponderaram-se os dados de ocorrência de presas pelos de abundância relativa de consumidores. Assim, os resultados do método Freqüência de Ocorrência (F.O.) foram associados aos de captura numérica $(\mathbf{N})$ por espécie, gerando:

a) o grau de participação relativa (P.R.) de cada item, segundo a população:

$$
\text { P.R.i,s }=\left(F . O_{i, i, s} \times N_{s}\right) \times 100 / \Sigma_{s=1 \rightarrow(}\left(F . O_{. i, s} \times N_{s}\right) \quad \text { e }
$$

b) o grau de participação global (P.G.) de cada item na assembléia:

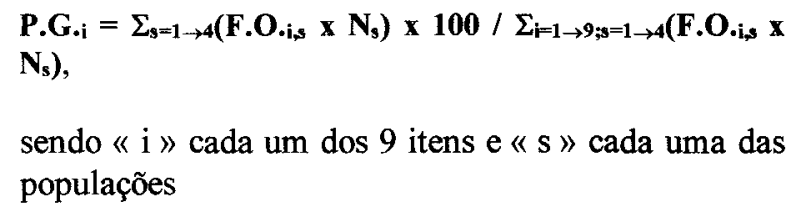

(C. arenaceus e C. spilopterus reunidas). 


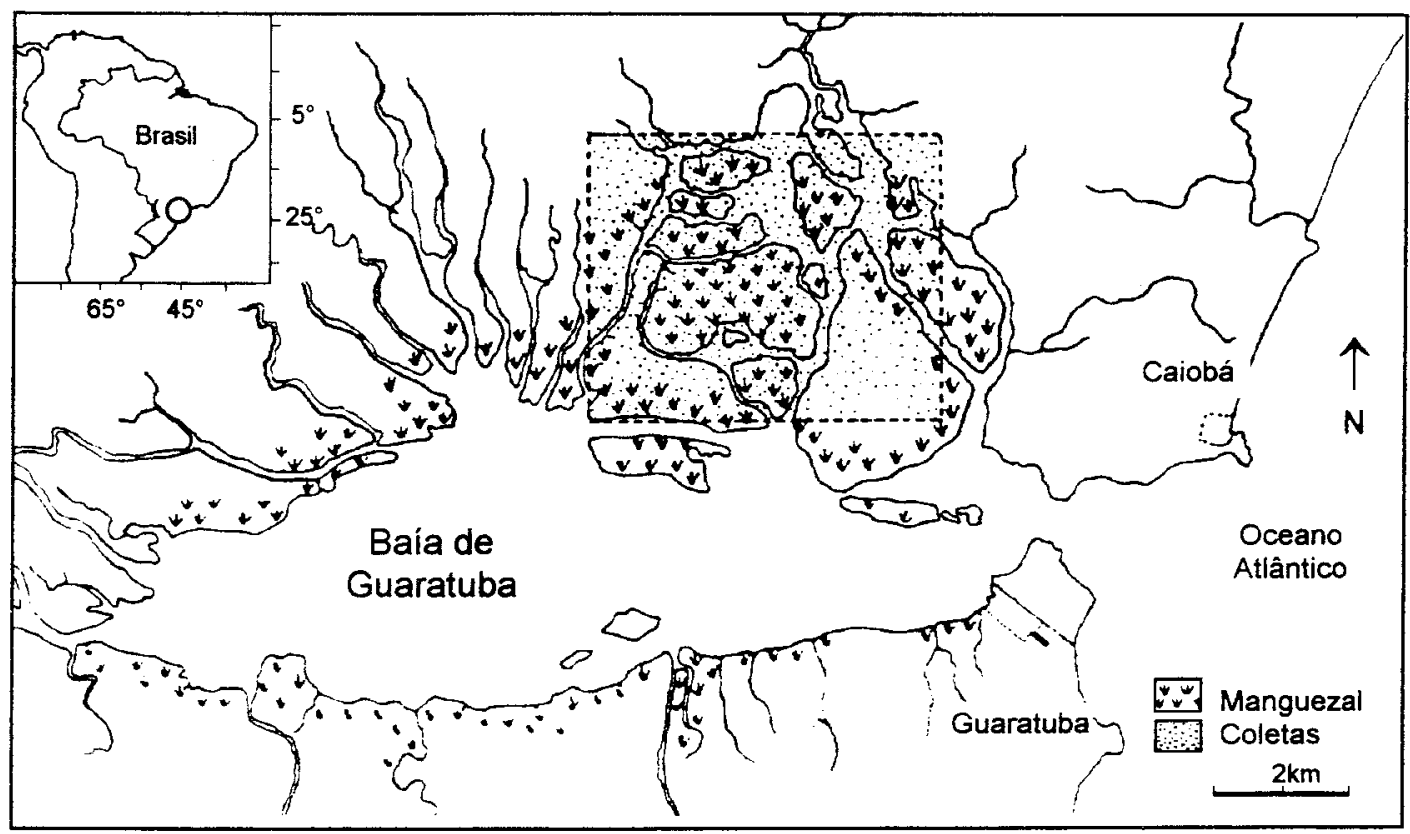

Fig. 1. Mapa indicativo da área estudada na Baía de Guaratuba, litoral do Estado do Paraná (2552'S;48³9'W). Pontos de coleta: variados trechos dos canais de mangue incluídos no retângulo tracejado. No detalhe, o círculo posiciona o sistema em relação à costa brasileira.

\section{Resultados}

Considerando o período inteiro de estudo, as duas espécies do gênero Citharichthys (comprimento: 62 a $190 \mathrm{~mm}$ ) compuseram cerca de $70 \%$ do valor total das capturas, representadas por uma abundância média mensal de 14,1 exemplares e $227 \mathrm{~g}$. E. crossotus ( 59 a $130 \mathrm{~mm}$ ) e $A$. lineatus $(77$ a
$174 \mathrm{~mm}$ ) posicionaram-se a seguir em densidade (respectivamente 2,4 e 2,2 exemplares), porém em biomassa esta posição foi partilhada entre $A$. lineatus e $S$. tessellatus (120 a $182 \mathrm{~mm}$ ), respectivamente $43 \mathrm{e}$ $40 \mathrm{~g}$ (Fig. 2). A densidade média mensal de $S$. tessellatus foi de 1,4 exemplares, e a biomassa média mensal de $E$. crossotus, $25 \mathrm{~g}$.

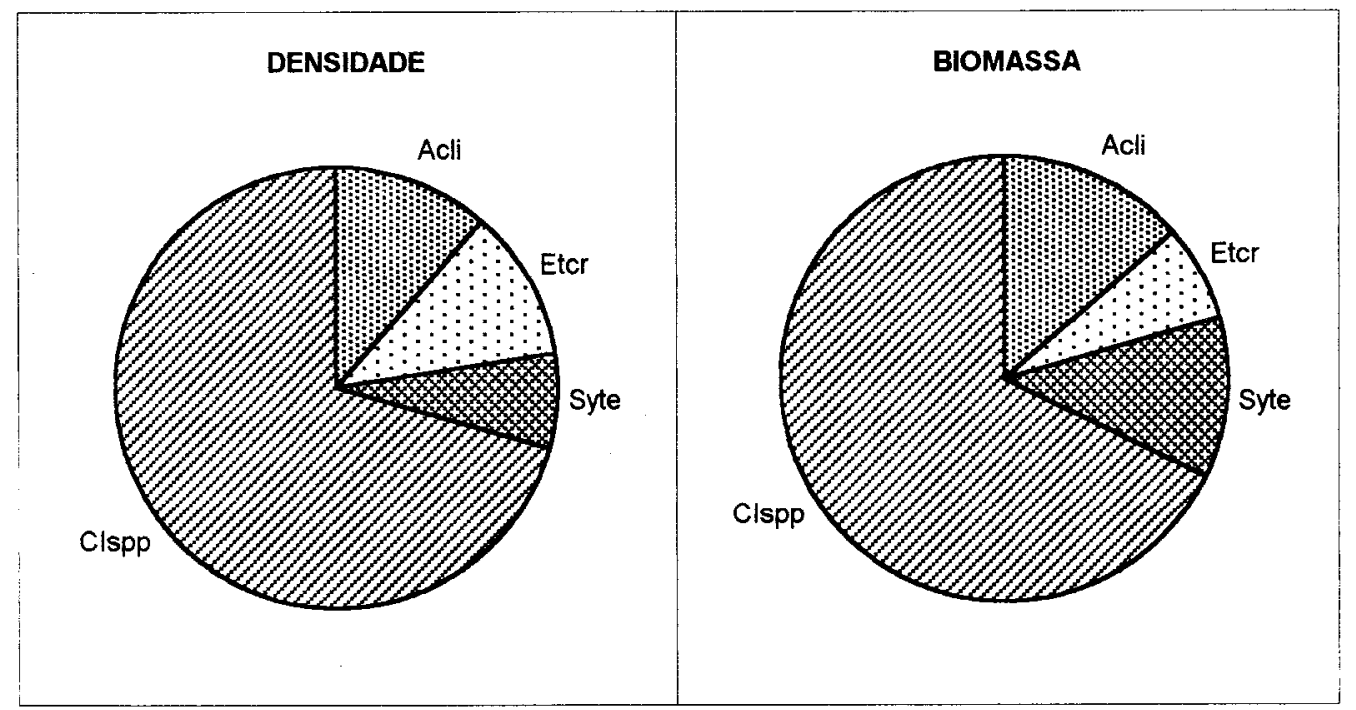

Fig. 2. Distribuição porcentual de densidade e de biomassa das capturas de linguados no manguezal de Guaratuba, entre outubro/93 e fevereiro/97, de acordo com a espécie: Acli: Achirus lineatus. Etcr: Etropus crossotus; Syte: Symphurus tessellatus, Clspp: dados reunidos de Citharichthys arenaceus e C. spilopterus. 
Quanto aos Índices de Preponderância do conteúdo estomacal, em $C$. arenaceus e $C$. spilopterus alcançaram maior valor os itens crustáceos Caridea (camarões) e peixes, conforme indicam tanto a análise dos dados conjuntos para o gênero, como a relativa a cada espécie (Tab. 1; Fig. 3). Ambas evidenciam, igualmente, a participação de moluscos Gastropoda, porém na análise conjunta das espécies o Índice de Preponderância deste item foi superado pelo de Brachyura (Fig. 3). Foram também registrados outros crustáceos (Amphipoda e Isopoda), moluscos Bivalvia e anelídeos Polychaeta, nenhum destes presente em mais que $10 \%$ dos indivíduos (Tab. 1).

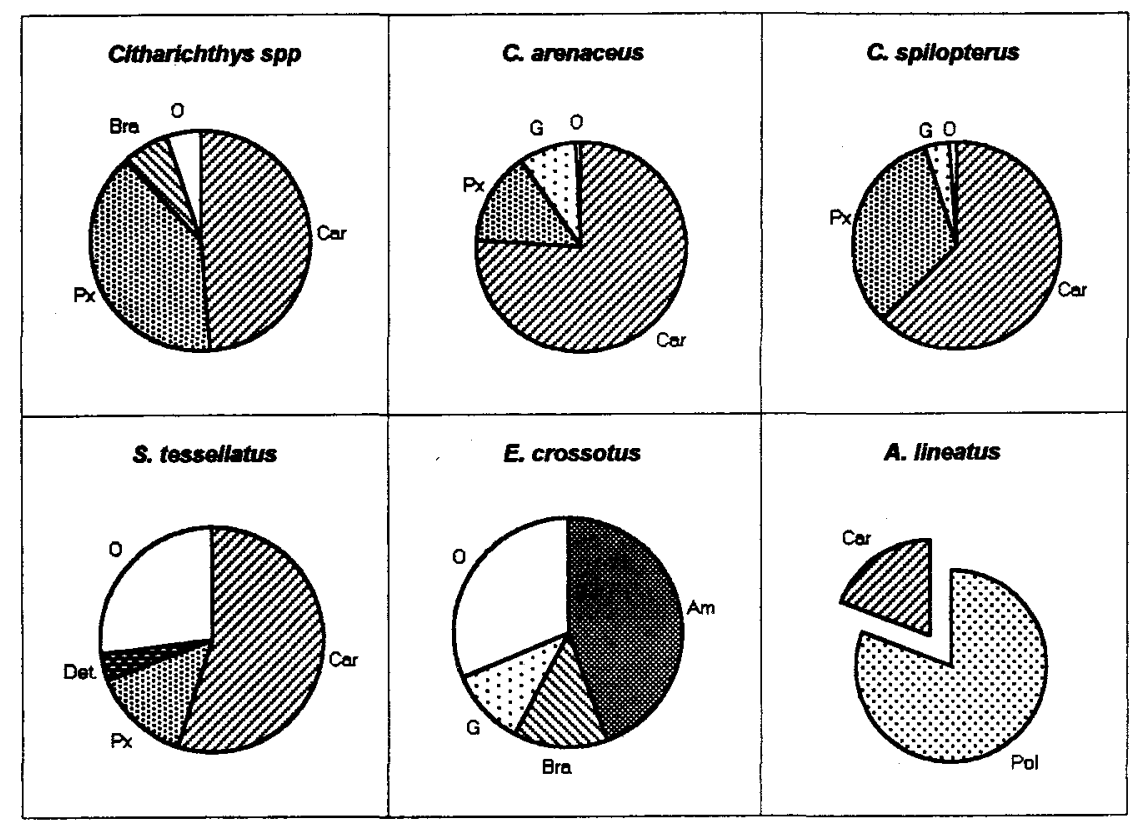

Fig. 3. Categorias com maior valor de Índice de Preponderância na análise de conteúdo estomacal. Citharichthys $s p p$ : dados reunidos de $C$. arenaceus e $C$. spilopterus referentes ao período outubro/93-setembro/96; $C$. arenaceus e C. spilopterus: outubro/96-fevereiro/97. Demais espécies: outubro/93-fevereiro/97. Am: Amphipoda Gammaridae. Bra: Brachyura. Car: Caridea. G: Gastropoda. Pol: Polychaeta. Px : peixes. Det.: detritos. O: outros itens e material não-identificado. Dados precisos constam nas Tabelas I e II.

Tabela 1. Participação relativa dos itens do conteúdo estomacal, segundo o método de análise, em 39 exemplares de Citharichthys arenaceus (Ca), comprimento entre 81 e $173 \mathrm{~mm}, 26$ de C. spilopterus (Cs), comprimento entre 90 e $158 \mathrm{~mm}$, e 168 das duas espécies reunidas (Cas), comprimento entre 65 e $174 \mathrm{~mm}$.

\begin{tabular}{|c|c|c|c|c|c|c|c|c|c|}
\hline \multirow[b]{2}{*}{ ITENS } & \multicolumn{3}{|c|}{$\begin{array}{c}\text { FREQÜÊ̂NCIA DE } \\
\text { OCORRÊNCIA }\end{array}$} & \multicolumn{3}{|c|}{$\begin{array}{l}\text { Contagem } \\
\text { de Pontos }\end{array}$} & \multicolumn{3}{|c|}{$\begin{array}{c}\text { ÍNDICE DE } \\
\text { PREPONDERÂNCLA }\end{array}$} \\
\hline & $\mathrm{Ca}$ & $\mathrm{Cs}$ & Cas & $\mathrm{Ca}$ & Cs & Cas & $\mathbf{C a}$ & $\mathrm{Cs}$ & $\begin{array}{c}\mathrm{Ca} \\
\mathrm{s}\end{array}$ \\
\hline Peixes & 38,5 & 46,1 & 45,2 & 21,8 & 37,5 & 33,0 & 14,0 & 32,5 & 39,3 \\
\hline Polychaeta & & & 0,6 & & & 0,2 & & & $<0,1$ \\
\hline Gastropoda & 43,6 & 26,9 & 20,2 & 12,1 & 7,7 & 7,1 & 8,8 & 3,9 & 3,8 \\
\hline Bivalvia & 2,6 & & 6,5 & 0,1 & & 0,8 & $<0,1$ & & 0,1 \\
\hline Caridea & 82,1 & 69,2 & 51,2 & 55,5 & 47,9 & 36,0 & 76,2 & 62,4 & 48,5 \\
\hline Brachyura & 2,6 & 11,5 & 19,6 & 1,8 & 5,0 & 13,4 & 0,1 & 1,1 & 6,9 \\
\hline Gammaridae & 10,2 & 3,8 & 5,7 & 0,9 & 0,5 & 1,0 & 0,2 & $<0,1$ & 0,2 \\
\hline Caprellidae & 2,6 & & 0,6 & 0,1 & & 0,5 & $<0,1$ & & $<0,1$ \\
\hline Isopoda & 5,1 & 3,8 & 3,6 & 0,4 & 0,4 & 0,7 & $<0,1$ & $<0,1$ & 0,1 \\
\hline Larvas de Caridea & 2,6 & & 1,8 & 0,8 & & 0,2 & $<0,1$ & & $<0,1$ \\
\hline Larvas Megalopa & 7,7 & 3,8 & 5,9 & 3,9 & 1,0 & 2,9 & 0,5 & $<0,1$ & 0,5 \\
\hline Outros Crustacea & & & 3,0 & & & 1,3 & & & 0,1 \\
\hline Vegetais & & & 4,1 & & & 0,7 & & & 0,1 \\
\hline Não identificados & 2,6 & & 4,8 & 2,6 & & 2,2 & 0,1 & & 0,3 \\
\hline TOTAL & - & - & - & 100 & 100 & 100 & 99,9 & 99,9 & 99,9 \\
\hline
\end{tabular}


Uma estreita semelhança foi verificada entre o conteúdo estomacal das espécies de Citharichthys e o de Symphurus tessellatus, que também apresentou elevadas participações de Caridea e peixes (Tab. 2; Fig. 3). Todavia, nesta última verificou-se uma expressiva ocorrência de detritos (fragmentos de origem animal ou vegetal, misturados a areia), além dos itens Polychaeta e Bivalvia em maior porcentagem que nas primeiras. Brachyura, Amphipoda e Gastropoda também foram registrados em $S$. tessellatus (Tab. 2).

Etropus crossotus e A. lineatus apresentaram resultados distintos entre si e daqueles das demais espécies. Em E. crossotus foram registrados principalmente Amphipoda da família Gammaridae e secundariamente Brachyura e Gastropoda (Tab. 2; Fig. 3). Detectaram-se ainda Amphipoda da família Caprellidae, Isopoda, Polychaeta e moluscos Bivalvia, todos em mais que $10 \%$ dos exemplares, e peixes e outros crustáceos em menos que $10 \%$ (Tab.
2). Em A. lineatus o conteúdo estomacal compôs-se essencialmente de Polychaeta e Caridea; o outro item presente - peixes - esteve representado em apenas $5 \%$ dos exemplares (Tab. 2; Fig. 3).

A análise dos valores de participação relativa (P.R.) dos diferentes itens na dieta das espécies revela que mais que $90 \%$ das ocorrências de peixes, assim como mais de $65 \%$ das de Caridea, Brachyura, Gastropoda e Bivalvia, referem-se às espécies de Citharichthys (Fig. 4). Os Amphipoda Gammaridae e Caprellidae encontram-se, em 60 a $80 \%$ dos casos, integrando a dieta de E. crossotus, e Polychaeta em $70 \%$ a de $A$. lineatus. Isopoda reparte-se em freqüências próximas a $50 \%$ entre Citharichthys spp e E. crossotus (Fig. 4). Na assembléia como um todo, Caridea e peixes apresentam os maiores valores de participação global (P.G.), seguidos de Gastropoda, Brachyura e Polychaeta (Fig. 5).

Tabela 2. Participação relativa dos itens do conteúdo estomacal, segundo o método de análise, em 36 exemplares de Etropus crossotus (Ec), comprimento entre 59 e $121 \mathrm{~mm}, 12$ de Symphurus tessellatus (St), comprimento entre 132 e 179 $\mathrm{mm}$, e 16 de Achirus lineatus (AI), comprimento entre 77 e $121 \mathrm{~mm}$.

\begin{tabular}{|c|c|c|c|c|c|c|c|c|c|}
\hline \multirow[b]{2}{*}{ ITENS } & \multicolumn{3}{|c|}{$\begin{array}{c}\text { FREQÜênCIA de } \\
\text { OCORRÊnCIA }\end{array}$} & \multicolumn{3}{|c|}{$\begin{array}{l}\text { Contagem } \\
\text { DE Pontos }\end{array}$} & \multicolumn{3}{|c|}{ Índice de PreponderânCla } \\
\hline & Ec & $\overline{\text { St }}$ & Al & Ec & St & Al & Ec & $\mathbf{S t}$ & Al \\
\hline Peixes & 5,5 & 33,3 & 5,2 & 1,4 & 15,8 & 0,1 & 0,3 & 14,3 & $<0,1$ \\
\hline Polychaeta & 16,7 & 16,7 & 78,9 & 10,7 & 8,3 & 71,6 & 5,9 & 3,8 & 80,8 \\
\hline Gastropoda & 33,3 & 25,0 & & 9,9 & 3,1 & & 10,7 & 2,1 & \\
\hline Bivalvia & 11,1 & 16,7 & & 3,0 & 2,5 & & 1,1 & 1,1 & \\
\hline Caridea & 8,3 & 58,3 & 47,4 & 5,8 & 34,6 & 28,3 & 1,6 & 54,8 & 19,1 \\
\hline Brachyura & 30,5 & 8,3 & & 13,1 & 8,3 & & 13,1 & 1,9 & \\
\hline Gammaridae & 58,3 & 8,3 & & 23,3 & 0,9 & & 44,5 & 0,2 & \\
\hline Caprellidae & 13,8 & & & 2,3 & & & 1,0 & & \\
\hline Isopoda & 16,7 & & & 7,3 & & & 3,9 & & \\
\hline Larvas Decapoda & 2,8 & & & 2,0 & & & 0,2 & & \\
\hline Outros Crustacea & 2,8 & & & 2,6 & & & 0,2 & & \\
\hline Vegetais & 8,3 & & & 1,7 & & & 0,5 & & \\
\hline Detritos & & 33,3 & & & 23,2 & & & 21,0 & \\
\hline Não identificados & 30,5 & 8,3 & & 16,9 & 3,3 & & 17,0 & 0,8 & \\
\hline TOTAL & - & - & - & 100 & 100 & 100 & 100 & 100 & 99,9 \\
\hline
\end{tabular}

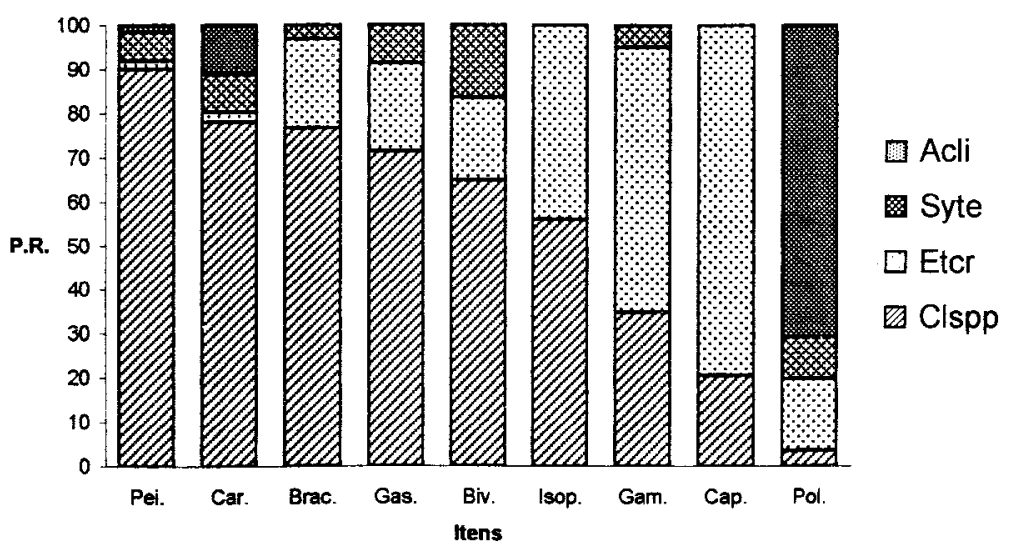

Fig. 4. Representação gráfica dos valores de participação relativa (P.R.) de 9 itens na dieta das espécies, relativamente à densidade de cada uma. Acli: A. lineatus. Syte: S. tessellatus. Etcr: E. crossotus. CIspp: Citharichthys spp. Pei.: peixes. Car.: Caridea. Brac.: Brachyura. Gas.: Gastropoda. Biv.: Bivalvia. Isop.: Isopoda. Gam.: Gammaridae. Cap.: Caprellidae. Pol.: Polychaeta. 


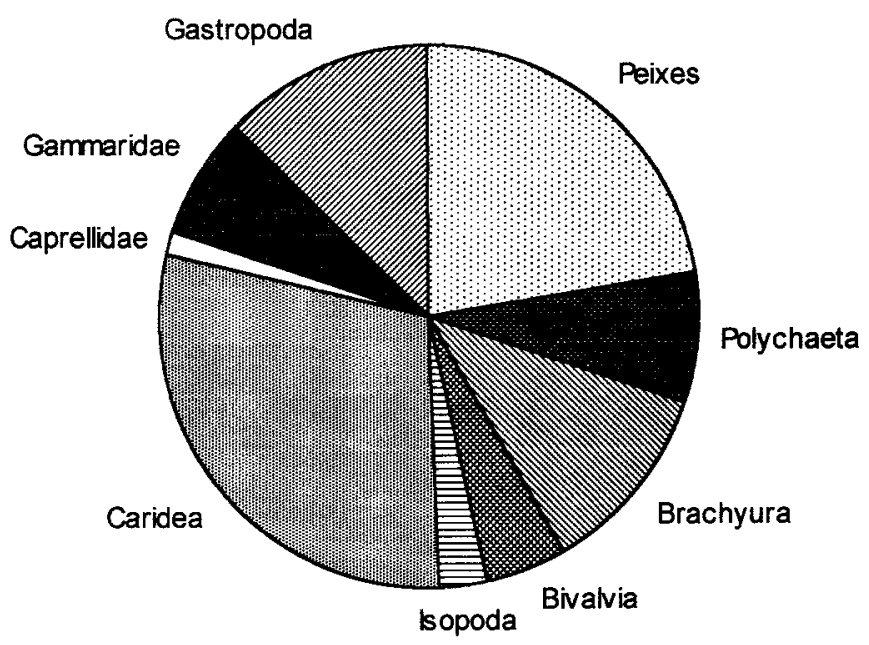

Fig. 5. Representação gráfica dos valores de participação global de 9 itens na dieta da assembléia, relativamente à densidade de cadá espécie.

\section{Discussão}

Inicialmente algumas observações de ordem metodológica devem ser formuladas. Diversos autores, especializados em estudos sobre alimentação de peixes, têm enfatizado as restrições que a análise de conteúdo estomacal oferece para a estimativa do verdadeiro regime alimentar de uma espécie. $\mathrm{Na}$ origem do problema destacam-se as diferentes velocidades de digestão, segundo a natureza do alimento, e as limitações para quantificação do volume ocupado por cada item (Zavala-Camin, 1996). Na presente investigação, as inferências são ainda mais restritivas em virtude (1) do reduzido número de exemplares analisados, (2) da ausência de um tratamento particular por estação do ano e faixa etária, e (3) da reunião das duas espécies de Citharichthys em um grupo único, exceto durante uma pequena parte do período. A circunstância número 2 , por exemplo, cuja influência sobre a interpretação da dieta de uma espécie é bem exemplificada por Soares (1989) e Rodriguez (1996), pode ser a causa da diferença constatada entre os dados referentes ao conjunto de $C$. arenaceus e $C$. spilopterus e aqueles referentes a uma e a outra, individualmente.

Como análise geral, constata-se que a dieta praticada pelos linguados no manguezal de Guaratuba segue o padrão já conhecido para o grupo, apoiado em peixes e invertebrados. No presente caso, estes últimos participam em maior grau, e, se for considerada a abundância relativa das populações dentro da assembléia, sobretudo através dos crustáceos Caridea, o item com mais alto valor de participação global (P.G.).
Possivelmente associado ao fato de o espectro ser amplo para a assembléia como um todo, particularidades existem segundo a espécie, e assim, com exceção de Citharichthys spp, algumas diferenças podem ser detectadas entre as populações. $S$. tessellatus e as duas espécies de Citharichthys parecem fazer uso de um semelhante elenco de presas. Entretanto, a ocorrência expressiva de detritos no conteúdo estomacal de $S$. tessellatus sugere a ingestão ativa de itens localizados mais próximo ao fundo, talvez em outra fração da coluna d'água em relação àqueles utilizados por $C$. arenaceus e $C$. spilopterus. Daí se entenderia igualmente a maior participação de Polychaeta e Bivalvia (normalmente restritos ao substrato) na primeira espécie em relação a estas duas, atenuando a possibilidade de sobreposição direta na utilização de recursos. Quanto a $E$. crossotus, trata-se de uma população que explora presas dos mesmos tipos que as espécies anteriores, todavia em proporções diferentes. Verificou-se que ela privilegia os itens de menor tamanho (Amphipoda), condizente com uma abertura bucal também menor que a de Citharichthys e de Symphurus. Depreende-se então que entre elas a sobreposição de dietas - se existe - é apenas parcial. E A. lineatus, finalmente, compõe a população cuja dieta se apresenta menos heterogênea. Em verdade, os itens ingeridos por esta espécie talvez se refiram a diferentes taxa de Polychaeta e de Caridea, podendo resultar ao final uma diversidade de presas maior que a de outros linguados (uma ressalva sobre o nível taxonômico investigado, aplicável a todas as populações). De qualquer forma, constata-se que a dieta de $A$. lineatus é a que envolve a menor variedade de categorias alimentares e também a menor sobreposição com as demais espécies. 
Finalmente, ao considerar a densidade das populações, o grau de participação relativa (P.R.) é mais um indicador do aproveitamento diferenciado dos recursos do meio: 3 unidades populacionais alcançam a posição de principal consumidora (mais que $60 \%$ das ocorrências) de pelo menos um dos itens analisados - Citharichthys (5 itens), E. crossotus (2) e A. lineatus (1).

Se os hábitos são diferentes entre as populações, estará a simpatria fundamentada em atributos originais de cada uma ou, ao contrário, em «adaptações locais»? Analisando-se os dados disponíveis para espécies congêneres, verifica-se que no litoral de São Paulo $\left(23^{\circ} \mathrm{S}\right)$ os principais componentes da dieta de Etropus longimanus e Symphurus jenynsi são os Polychaeta (Kawakami \& Amaral, 1983). Polychaeta, além de ofiuróides e crustáceos, são igualmente os itens mais consumidos por $S$. nigrescens no Mediterrâneo (MacPherson, 1978). Em Guaratuba, os Polychaeta respondem como principal componente alimentar apenas em $A$. lineatus. A diferença de hábitos entre aquelas populações e as de $E$. crossotus e $S$. tessellatus (que aqui utilizam principalmente crustáceos e peixes) pode estar associada a seus distintos ambientes de distribuição (mar aberto ou manguezal), cada um com seus próprios recursos e usuários a compartilhar. De fato, também o bagre Genidens genidens apresenta em Guaratuba um regime trófico diferente do praticado em certos sistemas lagunares não-manguezais, substituindo os peixes pelos crustáceos Brachyura como principal elemento da dieta (Chaves \& Vendel, 1996). Enfim, adaptações locais provavelmente existam, otimizando a utilização dos recursos do meio e contribuindo - no que concerne à alimentação - para a simpatria entre as espécies. Esta flexibilidade de hábitos, que na maior parte da assembléia estudada se materializa em dietas generalistas, conduz a sobreposições parciais entre as espécies, mas é considerada normal, necessária (Albaret, 1994) para que as populações lagunares resistam às fortes variações sazonais deste tipo de sistema.

\section{Agradecimentos}

Este trabalho foi concluído durante estágio Pósdoutorado do primeiro Autor junto ao Laboratoire d'Hydrobiologie Marine et Continentale da Université Montpellier II, França. Os autores agradecem, pois, aos Drs Gerard Lasserre e Jean-Luc Bouchereau (UM-II), por sua contribuição à Discussão. Também aos colegas Arno Blankensteyn e Edinalva Oliveira (UFPR), pelo auxilio na identificação de invertebrados; Mauricio Hostim
Silva e Silvia Helena Lucato (Univali), pela identificação de $S$. tessellatus; e ainda a dois Revisores anônimos, pelas oportunas críticas efetuadas sobre a primeira versão.

\section{Referências bibliográficas}

Albaret, J.-J. 1994. Les poissons, biologie et peuplements. In: Durand, J. R.; Dufour, P., Guiral, D. \& Zabi, S.G.F. eds. Environnement et ressources aquatiques de Côte-d'Ivoire. Tome II Les milieux lagunaires. Paris. ORSTOM, p.239279.

Chaves, P. T. C. \& Vendel, A. L. 1996. Aspectos da alimentação de Genidens genidens (Valenciennes) (Siluriformes, Ariidae) na Baía de Guaratuba, Paraná. Revta bras. Zool., 13(3):669-675.

Chaves, P. T. C. \& Vendel, A. L. 1997. Indicadores reprodutivos das espécies de Citharichthys Bleeker (Teleostei, Pleuronectiformes) na Baía de Guaratuba, Paraná, Brasil. Revta bras. Zool., 14(1):73-79.

Juras, A. A. \& Yamaguti, N. 1985. Food and feeding habits of king weakfish, Macrodon ancylodon (Bloch \& Schneider, 1801) caught in the Southern Coast of Brazil (Lat. $29^{\circ}$ to $32^{\circ}$ ). Bolm Inst. oceanogr., S Paulo, 33(2): 149-157.

Kawakami, E. \& Amaral, A. C. Z. 1983. Importância dos anelídeos poliquetos no regime alimentar de Etropus longimanus Norman, 1933 e Symphurus jenynsi Evermann \& Kendall, 1907 (Pisces, Pleuronectiformes). Iheringia, Ser. Zool., Porto Alegre, (62):47-54.

MacPherson, E. 1978. Regimèn alimentario de Symphurus nigrescens (Pisces, Cynoglossidae) en el Mediterraneo Occidental. Inv. Pesq., 42(2):325-333.

Pearcy, W. G. \& Hancock, D. 1978. Feeding habits of dover sole, Microstomus pacificus; rex sole, Glyptcephalus zachirus; slender sole, Lyopsetta exilis; and pacific sanddab, Citharichthys sordidus, in a region of diverse sediments and bathymetry off Oregon. Fish. Bull., 76(3):641651 .

Rodriguez, A. 1996. Régime alimentaire de deux Soleidae, Solea lascaris et Solea impar de la côte ouest de Bretagne. Cybium, 20(3):261-277. 
Soares, L. S. H. 1989. Alimentação de Isopisthus parvipinnis (Teleostei: Sciaenidae) na Baía de Santos, São Paulo. Bolm Inst. oceanogr., S Paulo, 37(2):95-105.

Toepfer, C. S. \& Fleeger, W. 1995. Diet of juvenile fishes Citharichthys spilopterus, Symphurus plagusia and Gobionellus boleosoma. Bull. mar. Sci, 56(1):238-249.

Zavala-Camin, L. A. 1996. Introdução aos estudos sobre alimentação natural em peixes. Maringá. EDUEM. 129p.

(Manuscrito recebido 20 agosto 1997; revisado 12 fevereiro 1998; aceito 15 abril 1998) 